\title{
Integrable Inhomogeneous Spin Chains in Generalized Lunin-Maldacena Backgrounds
}

\author{
Matheus Jatkoske Lazo \\ Centro Tecnológico de Alegrete, UFSM/Unipampa, \\ Alegrete, RS, Brazil. \\ Programa de Pós-graduação em Física, UFSM, 97111-900, \\ Santa Maria, RS, Brazil \\ lazo@smail.ufsm.br \\ (Received on 5 June, 2008)
}

\begin{abstract}
We obtain through a Matrix Product Ansatz the exact solution of the most general inhomogeneous spin chain with nearest neighbor interaction and with $U(1)^{2}$ and $U(1)^{3}$ symmetries. These models are related to the one loop mixing matrix of the Leigh-Strassler deformed $N=4$ SYM theory, dual to type IIB string theory in the generalized Lunin-Maldacena backgrounds, in the sectors of two and three kinds of fields, respectively. The solutions presented here generalizes the results obtained by the author in a previous work for homogeneous spins chains with $U(1)^{N}$ symmetries in the sectors of $N=2$ and $N=3$.
\end{abstract}

Keywords: Spin chains; Matrix product ansatz; Bethe ansatz; AdS/CFT

\section{INTRODUCTION}

String theory was first introduced in the 60's years, as a tentative to describe the big amount of mesons and hadrons discovered in particles accelerators. In this theory the particles are seen as different oscillations modes of the strings and it was able to describe some spectral characteristics of hadrons. Although this success, the original string theory is not able to explain many physical phenomenon mediated by strong interactions and nowadays the most successful theory to describe particle physics is the Quantum Cromodynamics (QCD). On the other hand, despite string theory has reemerged in the last three decades as a promising candidate for a quantum theory of all known interactions, its validity to describe nature has generated heated discussions since the theory is untestable due to the experimental impossibility to reach the tremendous energies found at the Plank scale. So, what is the actual relevance of string theory for real world physics? Either string theory is the correct theory to describe the world or not, remarkable resulties showed that string theory can be seen as a different formalism to quantum field theory. In QCD, it is not possible to obtain a satisfactory quantitative description in the small energy regime, when the coupling constant is very strong. In this regime, numerical calculation on the lattice is the best tool for study physical models. On the other hand, it was observed by 't Hooft [1] that the theory is simplified when the number $\mathrm{N}$ of colors is very high. 't Hooft derived a relationship between the topological structure of a Feynman graph and its $N$ dependence. When $1 / N$ is interpreted as a coupling constant, an expansion in $1 / N$ is similar to an expansion in a generic interacting string theory, resulting in a relation between strings and planar diagrams. If the $N=3$ can be regarded as a large $N$, it explain why the string models of 60 's years was able to give the right relation for some spectral characteristics of hadrons. More recently a remarkable result arouses new interest in the duality between string theory and quantum field theory. Maldacena conjectured that IIB string theory on the curved background $A d S_{5} \times S^{5}$ (anti-de Sitter and sphere spaces) should be equivalent to $N=4$ Super YangMills (SYM) [3-5]. This conjecture, AdS/CFT, relates opera- tors, states, correlation functions and dynamics of both theories. One of the most important results of this conjecture predicts that the spectrum of scaling dimension operator of gauge invariant operators, in the conformal field theory, should coincide with the spectrum of energies $E$ of string states. Furthermore, this correspondence relates the weak coupling constant regime, in the gauge theory, with the strong coupling constant ones, in the string theory.

The Maldacena's conjecture need yet to be fully proved and since the discovery of the relation between the planar dilatation operator of the $N=4 \mathrm{SYM}$ with an integrable so(6) quantum spin chains [2], integrability has played a prominent role in the exploration of the Maldacena's correspondence. The study of the planar dilatation operator's integrability is very important because it not only enable us to test the Maldacena's correspondence as it is an generator of nontrivial integrable models. Exactly solvable models are of interest in high energy physics, condensed matter physics, statistical mechanics and mathematics since the pioneering work of Hans Bethe [6] (see, e.g., [7-10] for reviews). According to this ansatz the amplitudes of the eigenfunction are expressed by a nonlinear combination of properly defined plane waves. On the other hand, in the last two decades several different ansatz were introduced in the literature under the general name of matrix product ansatz (MPA). The first formulation was done for the description of the ground-state eigenfunction of some special non-integrable quantum chains, the so called valence-bond solid models [11-14]. The MPA becomes also a successful tool for the exact calculation of the stationary probability distribution of some stochastic one dimensional systems [15-17]. An extension of this last MPA, called dynamical MPA was introduced in [18, 19] and extended in [20]. This last ansatz gives the time-dependent probability distribution for some exact integrable systems. The MPA we are going to use in this paper was introduced in [21-24]. This ansatz was applied with success in the evaluation of the spectra of several integrable quantum Hamiltonians [21-23], transfer matrices [2527] and the time-evolution operator of stochastic systems [24]. According to this ansatz, the amplitudes of the eigenfunctions are given in terms of a product of matrices where the matrices 
obey appropriated algebraic relations. In the case of the Bethe ansatz the spectral parameters and the amplitudes of the plane waves are fixed, apart from a normalization constant, by the eigenvalue equation of the Hamiltonian or transfer matrix. On the other hand, in the MPA the eigenvalue equation fixes the commutation relations of the matrices defining the ansatz. In such case the spectrum of the Hamiltonian or transfer matrix, and the corresponding eigenfunctions, can be computed in a purely algebraic way.

In the present paper we obtain through a MPA the exact solution of the most general inhomogeneous spin chain with nearest neighbor interaction and with $U(1)^{2}$ and $U(1)^{3}$ symmetries. This model is related to the one loop dilatation operator in deformed Lunin-Maldacena backgrounds [28] and conformal field theories with deformations [29]. The solutions presented here generalizes the results obtained for homoge- neous spins chains with $U(1)^{N}$ symmetries in the sectors of $N=2$ and $N=3[30,31]$. In this model the coupling interaction between neighbor sites are not a constant as it is in the homogeneous model studied in $[30,31]$.

\section{THE INHOMOGENEOUS SPIN CHAINS}

We consider here the most general inhomogeneous 3-state spin chain with nearest neighbor interaction, periodic boundary condition and $U(1)^{3}$ symmetry. The $U(1)^{3}$ symmetry imply that the Hamiltonian describing the time evolution of this spin chain conserves the number of states of each type. By denoting the basis of states at a given site as $|\alpha\rangle(\alpha=1,2,3)$, the Hamiltonian in a periodic lattice with $L$ sites takes the form

$$
H=\sum_{j=1}^{L}\left(\sum_{\alpha \neq \beta=1}^{3} \Gamma_{\beta \alpha}^{\alpha \beta}(j, j+1) E_{j}^{\beta \alpha} E_{j+1}^{\alpha \beta}+\sum_{\alpha, \beta=1}^{3} \Gamma_{\alpha \beta}^{\alpha \beta}(j, j+1) E_{j}^{\alpha \alpha} E_{j+1}^{\beta \beta}\right)
$$

where $E^{\alpha \beta}$ are $3 \times 3$ Wyel matrices with elements $\left(E^{\alpha \beta}\right)_{i, j}=$ $\delta_{\alpha, i} \delta_{\beta, j}(\alpha, \beta=1,2,3)$. While the first term in the right hand side of (1) acts over neighbor sites exchanging its configuration $|\alpha\rangle_{j} \otimes|\beta\rangle_{j+1} \rightarrow|\beta\rangle_{j} \otimes|\alpha\rangle_{j+1}$ with rate $\Gamma_{\beta \alpha}^{\alpha \beta}(j, j+1)$, the second one is a diagonal operator with weight $\Gamma_{\alpha \beta}^{\alpha \beta}(j, j+1)$. The Hamiltonian (1) in a particular case $\left(\Gamma_{\gamma \delta}^{\alpha \beta}(j, j+1)=\Gamma_{\gamma \delta}^{\alpha \beta}\right.$ constant) contain the homogeneous integrable spin-1 chain, related to the one loop dilatation operator in deformed LuninMaldacena backgrounds, studied by Frolov e Tseytlin [31] and generalized in [30]. The eigenfunctions for (1) can be construct as

$$
\left|\Psi_{L}\right\rangle=\sum_{\alpha_{1}, \ldots, \alpha_{L}}^{*} \Psi^{\alpha_{1} \ldots \alpha_{L}}\left|\alpha_{1}, \ldots, \alpha_{L}\right\rangle \quad\left(\alpha_{j}=1,2,3\right)
$$

where the symbol $(*)$ in the sum denotes the restriction to the sets $\left\{\alpha_{1}, \ldots, \alpha_{L}\right\}$ with the same number $n_{\alpha}$ of spins in configuration $\alpha$ and $\Psi^{\alpha_{1} \ldots \alpha_{L}}$ is a constant.

\section{THE MPA}

In order to formulate a MPA for the Hamiltonian (1), we make a one-to-one correspondence between configurations of spins and products of abstract matrices. This matrix product is construct by making a correspondence between a site $j$ in the chain with spin configuration $\alpha=1,2,3$ and a matrix $A_{j}^{(\alpha)}$. Our MPA asserts that the components of the amplitude of the eigenfunction $\Psi^{\alpha_{1} \ldots \alpha_{L}}$ in (2) are obtained by associating them to a products of these matrices $A_{j}^{(\alpha)}$. Actually $A_{j}^{(\alpha)}$ are abstract operators with an associative product. A well defined eigenfunction is obtained, apart from a normalization factor, if all the amplitudes are related uniquely. Equivalently, in the subset of words (products of matrices) in the algebra containing $n_{\alpha}\left(n_{1}+n_{2}+n_{3}=L\right)$ matrices $A_{j}^{(\alpha)}$ there exists only a single independent word. The relation between any two words gives the ratio between the corresponding amplitudes of the components of the eigenfunction $\left|\Psi_{L}\right\rangle$. To formulated the ansatz we can choose any uniform operation on the matrix products that gives a non-zero scalar to make a map between the amplitudes $\Psi^{\alpha_{1} \ldots \alpha_{L}}$ in (2) and a matrix product [? ]:

$$
\Psi^{\alpha_{1} \ldots \alpha_{L}} \Longleftrightarrow A_{1}^{\left(\alpha_{1}\right)} A_{2}^{\left(\alpha_{2}\right)} \cdots A_{L}^{\left(\alpha_{L}\right)} \quad\left(\alpha_{j}=1,2,3\right) .
$$

It is obvious that the 3 states $|\alpha \ldots \alpha\rangle(\alpha=1,2,3)$ are all eigenstates of the Hamiltonian (1). In the following we shall choose $|1 \ldots 1\rangle$ as our reference state. The Hamiltonian (1) when applied to the components of the eigenfunction (2) where we do not have spins configurations $|\alpha\rangle(\alpha=2,3)$ at nearest neighbor sites and at boundary positions give us the constraints, for the amplitudes $\Psi^{\alpha_{1} \ldots \alpha_{L}}$ (3), 


$$
\begin{aligned}
& \varepsilon_{n} A^{x_{1}-1} A_{x_{1}}^{\left(\alpha_{1}\right)} A^{x_{2}-x_{1}-1} \cdots A_{x_{j-1}}^{\left(\alpha_{j-1}\right)} A^{x_{j}-x_{j-1}-1} A_{x_{j}}^{\left(\alpha_{j}\right)} A^{x_{j+1}-x_{j}-1} A_{x_{j+1}}^{\left(\alpha_{j+1}\right)} \cdots A_{x_{n}}^{\left(\alpha_{n}\right)} A^{L-x_{n}} \\
& \quad=\sum_{j=1}^{n}\left[\Gamma_{1 \alpha_{j}}^{\alpha_{j} 1}(j, j+1) A^{x_{1}-1} A_{x_{1}}^{\left(\alpha_{1}\right)} A^{x_{2}-x_{1}-1} \cdots A_{x_{j-1}}^{\left(\alpha_{j-1}\right)} A^{x_{j}-x_{j-1}-2} A_{x_{j}-1}^{\left(\alpha_{j}\right)} A^{x_{j+1}-x_{j}} A_{x_{j+1}}^{\left(\alpha_{j+1}\right)} \cdots A_{x_{n}}^{\left(\alpha_{n}\right)} A^{L-x_{n}}\right. \\
& \left.\quad+\Gamma_{\alpha_{j+1} \alpha_{j+1}}^{1 \alpha_{j+1}}(j, j+1) A^{x_{1}-1} A_{x_{1}}^{\left(\alpha_{1}\right)} A^{x_{2}-x_{1}-1} \cdots A_{x_{j-1}}^{\left(\alpha_{j-1}\right)} A^{x_{j}-x_{j-1}} A_{x_{j}+1}^{\left(\alpha_{j}\right)} A^{x_{j+1}-x_{j}-2} A_{x_{j+1}}^{\left(\alpha_{j+1}\right)} \cdots A_{x_{n}}^{\left(\alpha_{n}\right)} A^{L-x_{n}}\right] \\
& \quad+\sum_{l=1}^{L}\left[\Gamma_{\alpha_{l} 1}^{\alpha_{l} 1}(l, l+1)+\Gamma_{1 \alpha_{l+1}}^{1 \alpha_{l+1}}(l, l+1)\right] A^{x_{1}-1} A_{x_{1}}^{\left(\alpha_{1}\right)} A^{x_{2}-x_{1}-1} \cdots A_{x_{n}}^{\left(\alpha_{n}\right)} A^{L-x_{n}} \quad\left(\alpha_{j}=1,2,3\right)
\end{aligned}
$$

where $\varepsilon_{n}$ is the energy of the eigenfunction (2), $A \equiv A_{x}^{(1)}, n=n_{2}+n_{3}$, and $x_{1}, \ldots, x_{n}$ are the position in the spin chain where we have a state configuration $|\alpha \neq 1\rangle$. A convenient solution of this last equation is obtained by identifying the matrices $A_{x}^{(\alpha)}$ $(\alpha=2,3)$ as composed by spectral-parameter-dependent matrices. The distinguibility of states configurations allows two types of solutions. The standard solution is obtained if each of the matrices $A_{x}^{(\alpha)}(\alpha=2,3)$ is composed of $n=n_{2}+n_{3}$ spectral parameter dependent matrices $[22,24,30]$. A second class of solutions is obtained if matrices $A_{x}^{(\alpha)}(\alpha=2,3)$ with different $\alpha$ value are composed of by distinct sets of spectral parameters matrices [22, 30]. Here we will consider only the standard solution but our model can be easily extended to the second class problem. In the present case, the matrices $A_{x}^{(\alpha)}(\alpha=2,3)$ can be written in terms of the matrix $A$ and $n=n_{2}+n_{3}$ spectral parameter dependent matrices $A_{x, k_{j}}^{(\alpha)}[?]$ :

$$
A_{x}^{(\alpha)}=\sum_{j=1}^{n} A_{x, k_{j}}^{(\alpha)} A, \quad(\alpha=2,3),
$$

where the matrices $A_{x, k_{j}}^{(\alpha)}$ satisfy the following commutation relations with the matrix $A$ :

$$
A_{x, k_{j}}^{(\alpha)} A=g_{\alpha}(x, x+1) e^{i k_{j}} A A_{x+1, k_{j}}^{(\alpha)}, \quad(\alpha=2, \ldots, N), \quad(j=1, \ldots, n),
$$

the parameters $k_{j}(j=1, \ldots, n)$ are in general complex numbers unknown a priori, and $g_{\alpha}(x, x+1)$ is a constant. The energy $\varepsilon_{n}$ is obtained by inserting (5) in (4), by using (6) and imposing that $\varepsilon_{n}$ is a symmetric function on the spectral parameters [? ]

$$
\varepsilon_{n}=\sum_{j=1}^{n}\left(\Gamma_{12}^{2} e^{1} e^{i k_{j}}+\Gamma_{22}^{12} e^{-i k_{j}}\right)+\sum_{\alpha=2}^{3} n_{\alpha}\left(\Gamma_{1 \alpha}^{1 \alpha}+\Gamma_{\alpha 1}^{\alpha} 1\right)+(L-2 n) \Gamma_{11}^{1} 1,
$$

where we need to impose

$$
g_{\alpha}(x, x+1)=\frac{\Gamma_{12}^{21}}{\Gamma_{1 \alpha}^{\alpha 1}(x, x+1)}=\frac{\Gamma_{\alpha 1}^{1 \alpha}(x, x+1)}{\Gamma_{21}^{12}} \quad(\alpha=2,3)
$$

and

$$
\Gamma_{11}^{11}(x, x+1)=\Gamma_{11}^{11}, \quad \Gamma_{1 \alpha}^{1 \alpha}(x, x+1)=\Gamma_{1 \alpha}^{1 \alpha}, \quad \Gamma_{\alpha 1}^{\alpha 1}(x, x+1)=\Gamma_{\alpha 1}^{\alpha 1} \quad(\alpha=2,3),
$$

where $\Gamma_{12}^{21}, \Gamma_{21}^{12}, \Gamma_{11}^{11}, \Gamma_{1 \alpha}^{1 \alpha}$ and $\Gamma_{\alpha 1}^{\alpha 1}$ are constants.

The relations coming from the eigenvalue equation for configurations where we have two spins configurations $|\alpha\rangle$ ( $\alpha=$ $2, \ldots, N)$ at nearest neighbor sites and are not located at boundary positions given us

$$
\begin{gathered}
\sum_{j, l=2}^{n}\left[\Gamma_{21}^{12}+\Gamma_{12}^{21} e^{i\left(k_{j}+k_{l}\right)}+\left(\Gamma_{\alpha 1}^{\alpha 1}+\Gamma_{1 \alpha}^{1 \alpha}-\Gamma_{11}^{11}-\Gamma_{\alpha \alpha}^{\alpha \alpha}(x, x+1)\right) e^{i k_{j}}\right] A_{y, k_{j}}^{(\alpha)} A_{y, k_{l}}^{(\alpha)}=0, \\
\sum_{j, l=2}^{n}\left[\Gamma_{21}^{12}+\Gamma_{12}^{21} e^{i\left(k_{j}+k_{l}\right)}+\left(\Gamma_{\alpha 1}^{\alpha 1}+\Gamma_{1 \beta}^{1 \beta}-\Gamma_{11}^{11}-\Gamma_{\alpha \beta}^{\alpha \beta}(x, x+1)\right) e^{i k_{j}}\right] A_{y, k_{j}}^{(\alpha)} A_{y, k_{l}}^{(\beta)}= \\
\sum_{j, l=2}^{n} \frac{\Gamma_{1 \alpha}^{\alpha 1}(x, x+1)}{\Gamma_{1 \beta}^{\beta 1}(x, x+1)} \Gamma_{\alpha \beta}^{\beta \alpha}(x, x+1) e^{i k_{l}} A_{y, k_{j}}^{(\beta)} A_{y, k_{l}}^{(\alpha)} \quad(\alpha \neq \beta),
\end{gathered}
$$


where we have used (3), (5)-(9) and $y=1, \ldots, L$. The relations (10) should be satisfied for all $x, y=1, \ldots L$. This is possible if we have constants $\Gamma_{\alpha \alpha}^{\alpha \alpha}, \Gamma_{\alpha \beta}^{\alpha \beta}, \Gamma_{\alpha \beta}^{\beta \alpha}$ and if

$$
\Gamma_{\alpha \alpha}^{\alpha \alpha}(x, x+1)=\Gamma_{\alpha \alpha}^{\alpha \alpha}, \quad \Gamma_{\alpha \beta}^{\alpha \beta}(x, x+1)=\Gamma_{\alpha \beta}^{\alpha \beta}, \quad \Gamma_{\alpha \beta}^{\beta \alpha}(x, x+1)=\frac{\Gamma_{1 \beta}^{\beta 1}(x, x+1)}{\Gamma_{1 \alpha}^{\alpha 1}(x, x+1)} \Gamma_{\alpha \beta}^{\beta \alpha} .
$$

Finally, the relations (10) fix the algebraic relations among the matrices $A_{x, k_{j}}^{(\alpha)}(\alpha=2,3)$ :

$$
A_{x, k_{j}}^{(\alpha)} A_{x, k_{l}}^{(\beta)}=\sum_{\alpha^{\prime}, \beta^{\prime}=2}^{3} S_{\beta^{\prime} \alpha^{\prime}}^{\alpha \beta}\left(k_{j}, k_{l}\right) A_{x, k_{l}}^{\left(\alpha^{\prime}\right)} A_{x, k_{j}}^{\left(\beta^{\prime}\right)}, \quad A_{x, k_{j}}^{(\alpha)} A_{x, k_{j}}^{(\beta)}=0 \quad(l \neq j=1, \ldots, n) .
$$

Relations (6) and (12) define completely the algebra whose structural constants are the $S$-matrix of the spin- $\frac{3}{2}$ model [21, 22, 30]. Since the several components of the wavefunction should be uniquely related, the above algebra should be associative. This associativity implies that the above $S$-matrix should satisfy the Yang-Baxter relations [7, 32], which is indeed the case [30]. The components of the wavefunction corresponding to the configurations where we have three or four particles in next-neigbouring sites would give in principle new relations involving three or four matrices $A_{x, k_{j}}^{(\alpha)}$. These new relations are however consequences of the above relations (6) and (12). It is important to mention that in the sector $n_{2}=0$ or $n_{3}=0$ (symmetry $\left.U(1)^{2}\right)$ the Hamiltonian (1) reduces to a inhomogeneous version of the well known asymmetric XXZ model [33].

In order to complete our solutions through the MPA (3) we should fix the spectral parameters, or momenta, $k_{1}, \ldots, k_{n}$. Theses parameter are fixed from the configurations where we have a spin configuration $\alpha=2,3$ at boundary positions $\left(x_{1}=1\right.$ or $\left.x_{n}=L\right)$. By using the algebraic relations (5), (6) and (12) we obtain the relation

$$
A_{1, k_{1}}^{\left(\alpha_{1}\right)} \cdots A_{n, k_{n}}^{\left(\alpha_{n}\right)} A^{L}=e^{i k_{j} L} \sum_{\alpha_{1}^{\prime}, \ldots, \alpha_{n}^{\prime}=2}^{N}\left\langle\alpha_{1}, \ldots, \alpha_{n}\left|\mathcal{T}^{(n)}\right| \alpha_{1}^{\prime}, \ldots, \alpha_{n}^{\prime}\right\rangle A_{1, k_{1}}^{\left(\alpha_{1}^{\prime}\right)} \cdots \alpha_{n, k_{n}}^{\left(\alpha_{n}^{\prime}\right)} A^{L},
$$

where we have used the identity (see [30])

$$
\sum_{\alpha_{j}^{\prime \prime}, \alpha_{j+1}^{\prime \prime}} S_{\alpha_{j}^{\prime} \alpha_{j}^{\prime \prime}}^{\alpha_{j} \alpha_{j+1}^{\prime \prime}}\left(k_{j}, k_{j}\right)=-1
$$

and

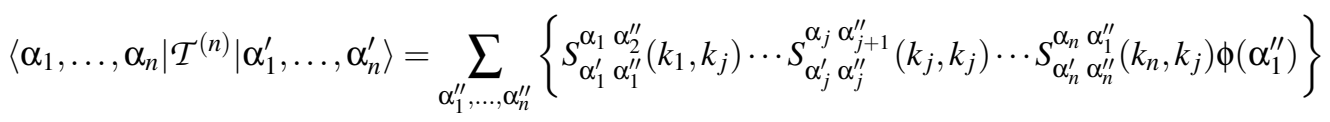

where $\phi\left(\alpha_{1}^{\prime \prime}\right)=\prod_{x=1}^{L} g_{\alpha_{1}^{\prime \prime}}(x, x+1), \quad$ is a $(2)^{n} \times(2)^{n}$ dimensional transfer matrix of an inhomogeneous vertex model (inhomogeneities $\left\{k_{l}\right\}$ ) with Boltzmann weights given by the $S$-matrix elements defined in (12). The model is defined on a cylinder of perimeter $n$ with a seam along its axis producing the twisted boundary condition

$$
S_{\alpha_{n}^{\prime} \alpha_{n}^{\prime \prime}}^{\alpha_{n} \alpha^{\prime \prime}}\left(k_{n}, k_{j}\right)=S_{\alpha_{n}^{\prime} \alpha_{n}^{\prime \prime}}^{\alpha_{n} \alpha_{n}^{\prime \prime}}\left(k_{n}, k_{j}\right) \phi\left(\alpha_{1}^{\prime \prime}\right) .
$$

Finally relation (13) with (16) give us the constraints for the spectral parameters:

$$
e^{-i k_{j} L}=\Lambda^{(n)}\left(k_{j},\left\{k_{l}\right\}\right) \quad(j=1, \ldots, n),
$$

where $\Lambda^{(n)}\left(k_{j},\left\{k_{l}\right\}\right)$ are the eigenvalues of the transfer matrix (15). The condition (17) leads to the problem of evaluation the eigenvalues of the inhomogeneous transfer matrix (15). This can be done through the algebraic Bethe ansatz [34] or the coordinate Bethe ansatz (see [35] and [36] for example).

\section{DISCUSSION AND CONCLUSIONS}

We solve through a MPA the most general inhomogeneous 3 -state spin chain with $U(1)^{3}$ symmetry and nearest neighbor interaction. We found that the coupling constants in (1) should satisfy the constraints (8), (9) and (11) in order to make the Hamiltonian (1) integrable. It is important to mention that different from the homogeneous spin chain, where the eigenstates (2) are also eigenstates of the translation operator due to the periodic boundary condition, in the inhomogeneous model the eigenstates do not have a defined momentum. It happens due to the inhomogeneities that broke the translational invariance of the system. The study of this new model can be of interest in the context of both AdS/CFT and condensed matter physics since it is related to the one loop dilatation operator in deformed Lunin-Maldacena backgrounds [28], conformal field theories with deformations [29] and inhomogeneous spin chains. Another quite interesting problem for the future 
concerns the formulation of the MPA for the case where we have open boundary conditions, as well as for quantum chains with no global conservation law such as the XYZ model, the 8 -vertex model or the case where the quantum chains are defined on open lattices with non-diagonal boundary fields.
Acknowledgements

This work has been supported by CAPES (Brazilian agencies).
[1] G. 't Hooft, Nucl. Phys. B 72, 461 (1974).

[2] J. A. Minahan and K. Zarembo JHEP 0303, 013 (2003).

[3] J. M. Maldacena Adv. Theor. Math. Phys. 2, 231 (1998).

[4] S. S. Gubser, I. R. Klebanov, and A. M. Polyakov, Phys. Lett. B 428, 105 (1998).

[5] E. Witten, Adv. Theor. Math. Phys. 2, 253 (1998).

[6] H. A. Bethe, Z. Phys. 71, 205 (1931).

[7] R. J. Baxter, 1982 Exactly Solved Models in Statistical Mechanics (New York: Academic).

[8] V. E. Korepin, A. G. Izergin, and N. M. Bogoliubov, 1992 Quantum Inverse Scattering Method, Correlation Functions and Algebraic Bethe Ansatz (Cambridge: Cambridge University Press).

[9] F. H. L. Essler and V. E. Korepin, 1994 Exactly Solvable Models of Strongly Correlated Electrons (Singapore: World Scientific).

[10] P. Schlottman, Int. J. Mod. Physics B 11, 355 (1977).

[11] I. Affleck, T. Kennedy, E. H. Lieb, and H. Tasaki, Commun. Math. Phys. 115, 477 (1988).

[12] D. P. Arovas, A. Auerbach, and F. D. M. Haldane, Phys. Rev. Lett. 60, 531 (1998)

[13] M. Fannes, B. Nachtergaele, and R. F. Werner, Commun. Math. Phys. 144, 443 (1992).

[14] A. Kluemper, A. Schadschneider, and J. Zittarz, Z. Phys. B 87, 281 (1992); Europhys. Lett. 24, 293 (1993).

[15] B. Derrida, Physics Reports 301, 65 (1988).

[16] B. Derrida, M. R. Evans, V. Hakim, and V. Pasquier, J. Phys. A: Math. Gen. 26, 1493 (1993).

[17] F. G. Alcaraz, S. Dasmahapatra, and V. Rittenberg, J. Phys. A: Math. Gen. 31, 845 (1998).

[18] R. B. Stinchcombe and G. M. Schütz, Phys. Rev. Lett. 75, 140 (1995).

[19] R. B. Stinchcombe and G. M. Schütz, Europhys. Lett. 29, 663 (1995).

[20] V. Popkov, M. E. Fouladvand, and G. M. Schütz, J. Phys. A: Math. Gen 35, 7187 (2002).

[21] F. C. Alcaraz and M. J. Lazo, J. Phys. A: Math. Gen. 37, L1 (2004).
[22] F. C. Alcaraz and M. J. Lazo, J. Phys. A: Math. Gen. 37, 4149 (2004).

[23] F. C. Alcaraz and M. J. Lazo, J. Phys. A: Math. Gen. 39, 11335 (2006).

[24] F. C. Alcaraz and M. J. Lazo, Braz. J. Phys. 33, 533 (2003).

[25] A. A. Ferreira and F. C. Alcaraz, Phys. Rev. E 74, P011115 (2006).

[26] M. J. Lazo, Physica A 374, 655 (2007).

[27] F. C. Alcaraz and M. J. Lazo, J. Stat. Mech. P08008 (2007).

[28] O. Lunin and J. Maldacena, JHEP 05, 033 (2005).

[29] R. G. Leigh and M. J. Strassler, Nucl. Phys. B 447, 95 (1995).

[30] M. J. Lazo, Braz. J. Phys. 38, 237 (2008).

[31] S. Frolov and A. A. Tseytlin, Nucl. Phys. B 668, 77 (2003).

[32] C. N. Yang, Phys. Rev. Lett. 19, 1312 (1968).

[33] C. N. Yang and C. P. Yang, Phys. Rev. 150, 321 (1966).

[34] P. P. Kulish and N. Yu Reshetikhin, Sov. Phys. -JETP 53, 108 (1981).

[35] F. C. Alcaraz and R. Z. Bariev, Braz. J. Phys. 30, 13 (2000).

[36] F. C. Alcaraz and R. Z. Bariev, Braz. J. Phys. 30, 655 (2000).

[37] N. Beisert, Phys. Rept. 405, 1 (2005).

[] Actually this uniform operation need to satisfy the symmetries of the model. In [30] we choose for this map the trace operation since the model were homogeneous and defined in a periodic chain. However in the present paper due to the inhomogeneities the trace operator do not work although we introduce another auxiliary matrix as in [21].

[] The most general relation $A_{x}^{(\alpha)}=\sum_{j=1}^{n} A^{a} A_{x, k_{j}}^{(\alpha)} A^{b}$ could be used. However (4) is more convenient since otherwise the $S$-matrix in (12) will depend on $a$ an $b$

[] There is another possibility to symmetrize the eigenvalue. It is to make all diagonal coupling equal $\Gamma_{\alpha \beta}^{\alpha \beta}(x, x+1)=\Gamma_{11}^{11}(x, x+$ 1) $(\alpha, \beta=1,2,3)$. In this case the diagonal interaction can be eliminated by adding a constant in the Hamiltonian (1) and the problem reduces to a free fermion model. 